Association for Information Systems

AIS Electronic Library (AISeL)

Wirtschaftsinformatik Proceedings 2005

Wirtschaftsinformatik

February 2005

\title{
Realisierung ubiquitärer Supply Networks auf Basis von Auto-ID- und Agenten-Technologien - Evolution oder Revolution?
}

Frank Teuteberg

Universität Osnabrück

Follow this and additional works at: http://aisel.aisnet.org/wi2005

\section{Recommended Citation}

Teuteberg, Frank, "Realisierung ubiquitärer Supply Networks auf Basis von Auto-ID- und Agenten-Technologien - Evolution oder Revolution?" (2005). Wirtschaftsinformatik Proceedings 2005. 1.

http://aisel.aisnet.org/wi2005/1

This material is brought to you by the Wirtschaftsinformatik at AIS Electronic Library (AISeL). It has been accepted for inclusion in Wirtschaftsinformatik Proceedings 2005 by an authorized administrator of AIS Electronic Library (AISeL). For more information, please contact elibrary@aisnet.org. 
In: Ferstl, Otto K, u.a. (Hg) 2005. Wirtschaftsinformatik 2005: eEconomy, eGovernment, eSociety; 7. Internationale Tagung Wirtschaftsinformatik 2005. Heidelberg: Physica-Verlag

ISBN: 3-7908-1574-8

(C) Physica-Verlag Heidelberg 2005 


\title{
Realisierung ubiquitärer Supply Networks auf Basis von Auto-ID- und Agenten-Technologien - Evolution oder Revolution?
}

\author{
Frank Teuteberg \\ Universität Osnabrück
}

\begin{abstract}
Zusammenfassung: Der Erfolg des Supply Chain Management (SCM) hängt entscheidend davon ab, dass relevante Informationen zeit- und kontextgerecht den Entscheidungsträgern zur Verfügung stehen. Nicht zuletzt aufgrund zunehmend kürzerer Produktlebenszyklen sind Lieferanten-Abnehmer-Netzwerke ständigen Veränderungen ausgesetzt. Bestehende Geschäftsprozesse müssen häufig an wechselnde Anforderungen und Partner angepasst werden. Dies führt zu einem hohen Aufwand für die Koordination von Aktivitäten innerhalb des Lieferanten-Abnehmer-Netzwerks. Mit der Agententechnologie und Auto-ID-Systemen (Auto-ID = Automatische Identifikation) auf der Basis von RFID (= Radio Frequency Identification) zum Tracking und Tracing von Ressourcen entlang von Lieferanten-Abnehmer-Netzwerken können wesentliche Beiträge zur Effektivierung des SCM geleistet werden. Im Rahmen dieses Beitrags wird der Status Quo der AgentenTechnologie zur Koordination von Lieferanten-Abnehmer-Netzwerken aufgearbeitet. Einsatzpotentiale eines agentenbasierten Tracking und Tracing auf der Basis von RFID werden dargestellt. Herausforderungen und zukünftige Entwicklungstrends im agentenbasierten SCM werden aufgezeigt. Eine Rahmenarchitektur eines Multi-Agenten-Systems (MAS) zur Koordination und Realisierung von ubiquitären Lieferanten-Abnehmer-Netzwerken wird vorgestellt.
\end{abstract}

Schlüsselworte: Auto-ID, Multi-Agenten-Systeme, RFID, Supply Chain Event Management, Tracking \& Tracing, Ubiquitäre Supply Networks

\section{Einleitung}

Die in der Praxis eingesetzten Planungssysteme zur Effektivierung des Supply Chain Management (SCM) werden dem Anspruch an eine zeitnahe Steuerung eines Lieferanten-Abnehmer-Netzwerks nur eingeschränkt gerecht, da bei kurzfristigen Änderungen jeweils ein neuer Gesamtplan generiert wird. Die Gesamtplanung ist somit störanfällig und inflexibel [Grol ${ }^{+} 01$, S. 143 ff; Dang ${ }^{+}$04, S. 10 ff]. Die derzeit auf dem Markt verfügbaren Planungssysteme eignen sich daher hauptsächlich für die mittel- und langfristige Planung von Leistungsprozessen entlang eines Lieferanten-Abnehmer-Netzwerks. Jedoch sind diese Systeme nicht a- 
daptiv und erfordern ein umfangreiches Customizing bei Änderungen des Lieferanten-Abnehmer-Netzwerks [Bode ${ }^{+}$01, S. 13 f].

Ziel des vorliegenden Beitrags ist es, den Status Quo der Agententechnologie zur informationstechnischen Koordination von Lieferanten-Abnehmer-Netzwerken darzustellen sowie Möglichkeiten zur Effektivierung des SCM, die aus dem Einsatz der Agententechnologie in Lieferanten-Abnehmer-Netzwerken resultieren können, aufzuzeigen. Dabei wird insbesondere auf die potentiellen Möglichkeiten eines agentenbasierten Tracking \& Tracing $(T \& T)$ entlang von LieferantenAbnehmer-Netzwerken auf der Basis von RFID eingegangen.

\section{Supply Networks}

Die Vernetzung der Unternehmen auf der Basis von Internet-Technologien sowie die zunehmende Auslagerung von betrieblichen Funktionen (Outsourcing) an externe Unternehmen wie z. B. 3rd Party Logistics Provider begünstigt die Entwicklung und den Ausbau von unternehmensübergreifenden Lieferanten-Abnehmer-Netzwerken. Nach einer Studie von Cap Gemini Ernst \& Young werden westeuropäische Unternehmen in den Jahren 2005 bis 200774 \% ihres LogistikBudgets für Leistungen externer Dienstleister verausgaben [Lang ${ }^{+}$02, S. 9].

Wertschöpfungsnetzwerke wie Lieferanten-Abnehmer-Netzwerke werden auch als Supply Chains bezeichnet [PaVa98, S. 1]. Aufgrund ihrer zumeist netzwerkartigen Struktur wird jedoch zunehmend der Begriff Supply Network bzw. Supply Web [Grol ${ }^{+} 01$, S. 143] verwendet. Der hohe Grad an Komplexität und die hohe Anzahl der Beziehungen zwischen den Unternehmen führt zu einem hohen Kommunikationsaufwand und erschwert es, kurzfristig entscheidungsrelevante Informationen beim Eintreten von Störereignissen bei den jeweiligen Netzwerkpartnern einzuholen. Supply Chain Event Management (SCEM) kann hierbei Abhilfe schaffen [Niss02].

SCEM ist ein Konzept zur unternehmensübergreifenden Erfassung, Überwachung und Bewertung von zufällig auftretenden Störereignissen entlang eines Supply Network. Im Vordergrund stehen der Informationsaustausch zwischen den von einem Störereignis (wie z. B. Verkehrsstau, Maschinenausfall) betroffenen Unternehmen sowie die schnelle Einleitung von Maßnahmen, um die Folgeauswirkungen der Störereignisse in Grenzen zu halten [Niss02]. Die Informationssammlung entlang des Supply Network kann durch sog. Tracking \& Tracing (T \& $\mathrm{T})$ erfolgen. Beim Konzept des $\mathrm{T} \& \mathrm{~T}$ zur Sendungsverfolgung erhebt das Tracking zeitpunktorientierte Daten zum jeweils aktuellen Status der untersuchten Ressource (z. B. Auftrag, Auslieferungsfahrzeug), während das Tracing zeitraumorientierte Tracking-Daten zu einer Historie (Zeitreihendaten) verdichtet und auf diese Weise langfristige Entwicklungen verdeutlicht. Die heutigen T \& T-Systeme beleuchten häufig nur einen Ausschnitt eines Supply Network, da sie häufig auf 
zentralen Konzepten basieren und die beteiligten Unternehmen nicht bereit sind, alle entscheidungsrelevanten Informationen ungefiltert an Partnerunternehmen entlang des Supply Network weiterzuleiten [Bode ${ }^{+} 01$, S. 15 f].

\subsection{Problemaspekte bei der Realisierung von Supply Networks}

Nachfolgend werden wesentliche Problemaspekte aufgeführt, mit denen die Beteiligten eines Supply Network konfrontiert werden $\left[\mathrm{Grol}^{+} 01\right.$, S. 143 f; KeKr04; ScHö03; Dang 02b; Dang ${ }^{+}$04, S. 19 f; Bode 01, S. 14]:

- Bullwhip-Effekt: Unter dem sog. Bullwhip-Effekt ist das Phänomen zu verstehen, dass signifikante Nachfrageschwankungen entlang der Wertschöpfungsstufen eines Supply Network zu beobachten sind, obwohl eine stabile Endnachfrage zu verzeichnen ist. Diese Nachfrageschwankungen resultieren aus einem Informationsdefizit der einzelnen Mitglieder eines Supply Network und führen letztendlich zu einem Koordinationsverlust, der mit einem Anstieg von Bestandskosten verbunden ist.

- Komplexität und Dynamik: Aufgrund immer kürzerer Produktlebenszyklen oder nur kurzfristig eingegangener Unternehmenskooperationen sind Supply Networks oftmals nur von temporärer Dauer und müssen daher häufig rekonfiguriert werden.

- Vertrauen und Kooperationsbereitschaft der Unternehmen: Mitglieder eines Supply Network sind oftmals Mitglieder mehrerer Supply Networks, die wiederum untereinander in Konkurrenz stehen können. Das gegenseitige Vertrauen der Mitglieder ist begrenzt, da ein zu großer Einfluss anderer Mitglieder auf das eigene Unternehmen bzw. Zugriff auf eigene Datenbestände mit Risiken verbunden ist. Mangelnde Kooperationsbereitschaft und Vertrauensverlust führen jedoch zu zunehmender Informationsintransparenz bzw. zum Zurückhalten entscheidungsrelevanter Informationen.

- Sicherheit: Sensible Unternehmensdaten, die per Internet oder sogar per Mobilfunk entlang des Supply Network übertragen werden, müssen vor Angriffen und Ausspähen durch Dritte durch geeignete Maßnahmen (wie z. B. Verschlüsselung oder digitale Signaturen) geschützt werden.

- Integrationsprobleme: Aufgrund von auf unterschiedlichen Datenformaten und Schnittstellen aufbauenden monolithischen Planungssystemen der einzelnen Netzwerkpartner wird zudem der überbetriebliche Informationsaustausch erschwert.

\subsection{Ubiquitäre Supply Networks}

Traditionelle Supply Chains sind den zuvor skizzierten Problemaspekten nicht mehr gewachsen. In Forschung und Praxis wird daher seit einigen Jahren die Rea- 
lisierung sog. adaptiver Supply Networks diskutiert. Im Rahmen dieses Beitrags wird der Begriff des ubiquitären Supply Network neu eingeführt. Tabelle 1 (in Anlehnung an [SAP02, S. 7]) führt die wesentlichen Charakteristika von "traditionellen" Supply Chains, adaptiven Supply Networks sowie ubiquitären Supply Networks auf.

\begin{tabular}{|c|c|c|c|}
\hline Charakteristika & $\begin{array}{l}\text { "Traditionelle" } \\
\text { Supply Chains } \\
\text { (Phase bis 2000) }\end{array}$ & $\begin{array}{l}\text { Adaptive Supply } \\
\text { Networks (Phase } \\
\text { ab 2000) }\end{array}$ & $\begin{array}{l}\text { Ubiquitäre Supply Net- } \\
\text { works (Phase ab 2006) }\end{array}$ \\
\hline $\begin{array}{l}\text { Informationsaus- } \\
\text { tausch }\end{array}$ & $\begin{array}{l}\text { Sequentiell + } \\
\text { langsam }\end{array}$ & $\begin{array}{l}\text { Parallel + dyna- } \\
\text { misch }\end{array}$ & Ubiquitär (“allgegenwärtig”) \\
\hline Planungshorizont & Tage/Wochen & Stunden/Tage & Minuten/Stunden \\
\hline Planung & $\begin{array}{l}\text { Batch-Betrieb/ } \\
\text { Zentraler Plan }\end{array}$ & Dynamisch & $\begin{array}{l}\text { Dynamisch/Portfolio an Al- } \\
\text { ternativplänen }\end{array}$ \\
\hline Antwortzeiten & Tage/Stunden & Stunden & Minuten \\
\hline Berechnungen & $\begin{array}{l}\text { Sammlung und } \\
\text { Berechnung histo- } \\
\text { rischer Daten }\end{array}$ & $\begin{array}{l}\text { Interpretation in } \mathrm{Re}- \\
\text { alzeit }\end{array}$ & $\begin{array}{l}\text { Interpretation gefilterter und } \\
\text { kontextsensitiver Daten in } \\
\text { Realzeit }\end{array}$ \\
\hline Steuerung & Zentral & Verteilt & Verteilt \\
\hline Event Management & Zentral/Manuell & Dynamisch/Verteilt & $\begin{array}{l}\text { Dynamisch/Verteilt/ } \\
\text { Fokus auf kritische Ausnah- } \\
\text { men }\end{array}$ \\
\hline Integration & Insellösungen & $\begin{array}{l}\text { Unternehmensüber- } \\
\text { greifend }\end{array}$ & Unternehmensübergreifend \\
\hline Standardisierung & Proprietär & Offen & Offen \\
\hline Zugriff & $\begin{array}{l}\text { Applikationen/ } \\
\text { Web-Browser }\end{array}$ & $\begin{array}{l}\text { Web-Browser/ Ap- } \\
\text { plikationen }\end{array}$ & $\begin{array}{l}\text { Multimodal (Web, Mobil, } \\
\text { Voice, externe Applikatio- } \\
\text { nen) }\end{array}$ \\
\hline $\begin{array}{l}\text { Rückkopplung und } \\
\text { Lernen }\end{array}$ & $\begin{array}{l}\text { Keine Rückkopp- } \\
\text { lung/kein Lernen }\end{array}$ & $\begin{array}{l}\text { Automatische Rück- } \\
\text { kopplung und Ler- } \\
\text { nen }\end{array}$ & $\begin{array}{l}\text { Automatische Rückkopplung } \\
\text { und kontinuierliches Lernen }\end{array}$ \\
\hline $\begin{array}{l}\text { Auto-ID auf der } \\
\text { Basis von RFID }\end{array}$ & Nicht eingesetzt & In Teilbereichen & Unternehmensübergreifend \\
\hline Basis-Technologien & $\begin{array}{l}\text { Planungssysteme } \\
\text { in Teilbereichen }\end{array}$ & Agenten & $\begin{array}{l}\text { Agenten, Web Services, } \\
\text { RFID, VoiceXML, Semantic } \\
\text { Web, P2P-Netzwerke, Mo- } \\
\text { bile Computing }\end{array}$ \\
\hline
\end{tabular}

Tabelle 1: Traditionelle, adaptive und ubiquitäre Supply Networks

Traditionelle Supply Chains sind insbesondere durch eine zentrale Steuerung und Inflexibilität gekennzeichnet. Adaptive Supply Networks zeichnen sich dagegen durch Flexibilität und Lernfähigkeit aus. Ubiquitäre Supply Networks ermöglichen darüber hinaus zu jeder Zeit und an jedem Ort einen multimodalen Zugriff (Voice, Web, mobil) auf entscheidungsrelevante Daten, die in Realzeit gefiltert den jeweiligen Entscheidungsträgern zur Verfügung gestellt werden.

Im Hinblick auf den Untertitel des vorliegenden Beitrags „Evolution oder Revolution?“ sind nach Auffassung des Autors ubiquitäre Supply Networks die nächste konsequente Evolutionsstufe nach adaptiven Suppy Networks. Sie erweitern diese insbesondere um die Möglichkeiten von Web Services, des Mobilfunks sowie der Auto-ID-Technologie RFID zum ubiquitären Informationszugriff und -austausch. 


\section{Basistechnologien zur Realisierung ubiquitärer Supply Networks}

\subsection{Softwareagenten und Multi-Agenten-Systeme}

In der Literatur existieren unterschiedliche Definitionen von Softwareagenten [Woo199, S. 25 f]. Dies liegt vor allem in der Vielzahl unterschiedlicher Anwendungsgebiete begründet. Nach einer Definition von Maes sind Softwareagenten „....computational systems that inhabit some complex dynamic environment, sense and act autonomously in this environment, and by doing so realize a set of goals or tasks for which they are designed " [Maes95, S. 108]. Häufig werden verschiedene Charakteristika zur Abgrenzung von Softwareagenten zu anderen Softwareparadigmen herangezogen. Ein Software-Agent ist demnach eine SoftwareEinheit, die insbesondere mehrere der folgenden Charakteristika aufweist: Interaktions- und Kommunikationsfähigkeit, Autonomie, Proaktivität, Mobilität, Intelligenz, Rationalität sowie Adaptivität [Wool99].

Softwareplattformen, auf denen mehrere autonome Software-Agenten als eigenständige Einheiten miteinander kommunizieren und kooperieren werden als MultiAgenten-Systeme (MAS) bezeichnet [Ferb99]. MAS bieten die Möglichkeit zur Konzeptualisierung und Modularisierung. Auf der Basis der aufgeführten Charakteristika ist somit eine realitätsnahe Modellierung von verteilten Problemlösungsprozessen in Supply Networks möglich [Grol ${ }^{+} 01$, S. 144 f], indem die einzelnen Entitäten eines Supply Network wie bspw. Maschinen, Auslieferungsfahrzeuge, Kunden, Aufträge, etc. durch Softwareagenten repräsentiert werden.

Einführungen zu den Forschungsgebieten Softwareagenten und MAS finden sich bspw. in [Ferb99; Woo199].

\subsection{Auto-ID-Infrastruktur}

Gemeinsam mit mehr als 100 Untenehmen aus der produzierenden Industrie, der IT sowie Dienstleistern und Handelskonzernen (wie z. B. IBM, SUN, Gilette, Wal-Mart) hat das Auto-ID Center, eine unabhängige Non-Profit-Organisation mit Hauptsitz in Massachussets, von 1999 bis Oktober 2003 an der Entwicklung von Industriestandards und Technologien zum nahtlosen Tracking und Tracing von Ressourcen entlang von Supply Networks gearbeitet. Nach der Überführung des Auto-ID Center in die EPCglobal Organization (www.EPCglobalinc.org) sowie die Auto-ID Labs (http://www.Auto-IDlabs.org), einem Forschungsverbund von 6 führenden Universitäten (MIT, University of Cambridge, University of Adelaide, Keio University, Fudan University und Universität von St. Gallen) auf dem Gebiet Auto-ID, werden derzeit die Arbeiten des Auto-ID Center von der EPCglobal Organization und den Auto-ID Labs nahtlos fortgesetzt. 
Eine Auto-ID-Infrastruktur zum T \& T von Objekten entlang von Supply Networks setzt sich aus den nachfolgenden Komponenten zusammen [Melo03]:

- RFID-Tags und Lesegeräten: RFID-Tags sind wenige Quadratmillimeter große Datenträger, die mit einer integrierten Antenne versehen per Funk innerhalb einer Distanz von bis zu einigen Metern durch spezielle Lesegeräte, sog. RFID-Reader, fernabgefragt werden können. Gegenüber traditionellen AutoID-Systemen auf der Basis von Barcode bieten RFID-Tags u. a. den Vorteil, dass bei einer schnelleren Datenübertragung wesentlich mehr Informationen zu mehreren Objekten gleichzeitig (sog. „Multitagging“) ausgelesen und gespeichert werden können und zudem kein direkter Kontakt zwischen Lesegerät und RFID-Tag erforderlich ist.

- EPC: Der EPC (= Electronic Product Code) dient zur eindeutigen Identifikation beliebiger Objekte der physischen Welt und erlaubt auf der Basis einer 96-Bit Darstellung die eindeutige Vergabe von über 68 Milliarden Seriennummern (z. B. für 268 Millionen Hersteller, die jeweils über 16 Millionen Produkte verfügen). Der EPC setzt sich wie folgt zusammen (vgl. Abbildung 1):

1. Header (8 Bit): Spezifiziert die EPC-Versionsnummer,

2. EPC Manager (28 Bit): Spezifiziert den Namen des Anbieters (i. d. R. der Hersteller eines Produkts),

3. Object Class (24 Bits): Spezifiziert die Objektklasse (bei Produkten typischerweise die Lagerhaltungseinheit),

4. Serial Number Field (36 Bits): Spezifiziert die eindeutige Identifikationsnummer des Objekts .

\subsection{B87.00016G.000168DB0}

Header EPC Manager Object Class Serial Number

Abbildung 1: Electronic Product Code (96 Bit)

- Object Name Service (ONS): Mittels ONS-Server werden die per RFID identifizierten EPC-Nummern der jeweiligen Objekte über IP-Adressen den auf einem Server hinterlegten Beschreibungen der Objekte zugeordnet.

- Physical Markup Language/PML-Server: PML (Physical Markup Language) ist eine XML-basierte Auszeichnungssprache zur Beschreibung physischer Objekte [Floe $\left.{ }^{+} 03\right]$. Bspw. können Informationen wie Produktzusammensetzung, Losnummer, Herstellungsdatum, etc. in PML beschrieben und auf einem PML-Server hinterlegt werden.

- Savant: Savant ist „Middleware-Software“, die quasi als „zentrales Nervensystem“ der Auto-ID-Architektur fungiert. Die Middleware-Software inter- 
agiert mit Lesegeräten, externen Applikationen sowie ONS- und PML-Server mittels spezieller Schnittstellen.

- Sensor-Technologien: Optional können thermische, akustische, magnetische, seismische oder auf der Infrarot-Technologie basierende Sensoren zum Einsatz kommen. Die Sensoren können dann bspw. per RFID-Technologie zusätzlich zu allgemeinen Produktdaten Informationen zur Temperatur, dem Geräuschpegel, Erschütterungen, Maße oder Geschwindigkeiten von Objekten per Mobilfunk übermitteln.

Folgende Schritte werden typischerweise bei der automatischen Identifikation von Ressourcen entlang von Supply Networks auf Basis der RFID-Technologie durchlaufen (vgl. Abbildung 2):

1. Electronic Product Code ist in einen RFID-Tag einzubetten, der wiederum an einem physischen Objekt (z. B. an einem T-Shirt) angebracht wird.

2. RFID-Reader scannen den entsprechenden RFID-Tag und senden den EPC zu einem Savant-Server.

3. Der Savant-Server sendet eine Anfrage an die Datenbank eines ONS-Server. Der ONS-Server ordnet den EPC einer IP-Adresse zu (sog. „Mapping“), die auf alle relevanten Informationen (in PML) zum entsprechenden Objekt verweist und sendet diese zurück an den Savant-Server.

4. Die entsprechenden Informationen wie z. B. Produktdaten, Hersteller, Abnehmer sowie andere relevante Daten sind auf einem PML-Server hinterlegt und werden in PML nach Übermittlung der URL an den Savant-Server zurück übertragen.

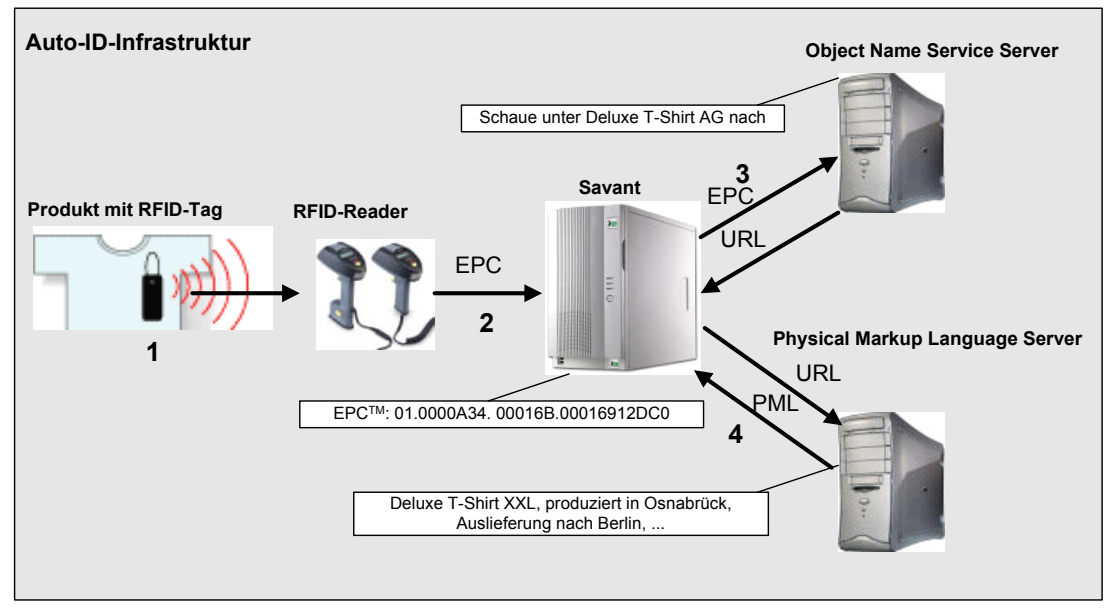

Abbildung 2: Auto-ID-Infrastrukur [In Anlehnung an: Melo03, S. 7] 


\section{Einsatzgebiete der Agententechnologie in Supply Networks - Status Quo}

Im Rahmen einer Literaturrecherche für diesen Beitrag wurde untersucht, in welchen Bereichen MAS im SCEM zum Einsatz kommen können und welche Herausforderungen auf dem Weg zur Realisierung ubiquitärer Supply Networks noch zu lösen sind. Einsatzgebiete von MAS im SCEM sind insbesondere die folgenden [Grol ${ }^{+} 01$, S. 147]:

- Beschaffungslogistik: Im Bereich der Beschaffungslogistik und elektronischer Märkte können Agenten im Auftrag der Marktteilnehmer Informationen beschaffen, Geschäfte anbahnen sowie Angebot und Nachfrage zusammenführen.

- Produktionslogistik: Im Bereich der Produktionslogistik können Agenten die verteilte Planung in der Fertigung unterstützen. Eine gemeinsame Lösungsfindung wird i. d. R. durch Kooperation der planenden Agenten untereinander gesteuert.

- Distributionslogistik: Im Bereich der Distributionslogistik können Agenten eingehende Aufträge disponieren. Ziel ist zumeist, zum einen die gesamte zugrunde liegende Wegstrecke der Auslieferungsfahrzeuge zu minimieren und zum anderen die Anzahl der Leerfahrten zu reduzieren.

- Tracking und Tracing: Im Bereich des T \& T können Agenten die Überwachung von geplanten und ungeplanten Ereignissen (Supply Chain Event Management) unterstützen sowie auf der Basis von RFID die Auslieferung von Produkten verfolgen (Sendungsverfolgung) und Handlungsempfehlungen beim Eintreten von Störereignissen geben.

- Unternehmensübergreifende Supply Networks: Im Bereich der Supply Networks können Agenten die Koordination des unternehmensübergreifenden Informationsaustausches zwischen den beteiligten Unternehmen unterstützen.

Eine Auswahl der in der Literatur beschriebenen MAS-Implementierungen bzw. Rahmenarchitekturen zur Effektivierung von Supply Networks bzw. isolierter Teilbereiche kann Tabelle 2 entnommen werden. Zur Klassifizierung der identifzierten MAS wurden die im Hinblick auf die Realisierung von ubiquitären Supply Networks (vgl. Tabelle 1) wichtigen Charakteristika „,multimodaler Zugriff“, Integration von Web Services zum Datenaustausch, Abstützung auf Peer-to-Peer (P2P)-basierte Netzwerkstrukturen verteilter Agentenplattformen und T \& $\mathrm{T}$ ergänzt. In der Spalte „Typ“ wird klassifiziert, ob es sich um einen Forschungsprototypen (FP) oder ein MAS handelt, das bereits über die Phase eines Forschungsprototypen hinaus ist und sich bereits im Praxiseinsatz (PE) befindet.

Chronologisch betrachtet zeigt sich (vgl. Tabelle 2, Spalte Autoren/Jahr), dass im Bereich des SCEM Agentensysteme seit Beginn der neunziger Jahre zunächst in den Bereichen Produktionslogistik und Distributionslogistik eingesetzt wurden. 
Agentenbasierte elektronische Märkte sind Mitte der neunziger Jahre zum Forschungsgegenstand geworden. Anschließend folgten Ende der 90er Jahre Arbeiten im Bereich unternehmensübergreifender Supply Networks. Forschungsarbeiten zur Realisierung agentenbasierter, ubiquitärer Supply Networks, insbesondere unter Einsatz von Auto-ID-Technologien (RFID), stehen dagegen noch ganz am Anfang und rücken erst in den letzten drei Jahren in den Mittelpunkt der Forschung.

\begin{tabular}{|c|c|c|c|c|c|c|c|}
\hline Domäne & MAS/Projekt & Тур & P2P & $\begin{array}{l}\text { Mobiler/ } \\
\text { Sprach- } \\
\text { basierter } \\
\text { Zugriff }\end{array}$ & $\begin{array}{c}\text { Inte- } \\
\text { gration } \\
\text { von } \\
\text { Web } \\
\text { Services }\end{array}$ & $\begin{array}{c}\text { T\&T } \\
\text { mittels } \\
\text { RFID }\end{array}$ & $\begin{array}{l}\text { Autoren/ } \\
\text { Jahr }\end{array}$ \\
\hline \multirow{2}{*}{$\begin{array}{l}\text { Beschaf- } \\
\text { fungslogistik }\end{array}$} & MAGMA & FP & Nein & Nein & Nein & Nein & [Tsve $^{+}$97] \\
\hline & Kasbah & $\mathrm{FP}$ & Nein & Nein & Nein & Nein & [ChMa96] \\
\hline \multirow{2}{*}{$\begin{array}{l}\text { Produktions- } \\
\text { logistik }\end{array}$} & MARTIN & FP & Nein & Nein & Nein & Nein & [Hens98] \\
\hline & Planet-AS & FP & Nein & Nein & Nein & Nein & [Mann95] \\
\hline \multirow{2}{*}{$\begin{array}{l}\text { Distributi- } \\
\text { onslogistik }\end{array}$} & MAS-MARS & FP & Nein & Nein & Nein & Nein & {$\left[\mathrm{Fisc}^{+} 95\right]$} \\
\hline & TRAMPAS-Z & FP & Nein & Nein & Nein & Nein & [Falk95] \\
\hline \multirow{3}{*}{$\begin{array}{l}\text { Tracking \& } \\
\text { Tracing }\end{array}$} & PAMAS & $\mathrm{FP}$ & $\mathrm{Ja}$ & Nein & Nein & $\mathrm{Ja}$ & [ZiPa03] \\
\hline & DIALOG & $\mathrm{PE}$ & $\mathrm{Ja}$ & Nein & Nein & $\mathrm{Ja}$ & {$\left[\right.$ Främ $\left.^{+} 03\right]$} \\
\hline & PROVE & FP & Nein & Nein & Nein & Nein & [Szir02] \\
\hline \multirow{4}{*}{$\begin{array}{l}\text { Unterneh- } \\
\text { mensüber- } \\
\text { greifende } \\
\text { Supply Net- } \\
\text { works }\end{array}$} & CoagenS & $\mathrm{PE}$ & $\mathrm{Ja}$ & Nein & $\mathrm{Ja}$ & Nein & [Dang $^{+}$02b] \\
\hline & DISPOWEB & $\mathrm{FP}$ & Nein & Nein & Nein & Nein & {$\left[\right.$ Groli $\left.^{+} 01\right]$} \\
\hline & Macroscope & $\mathrm{FP}$ & Nein & Nein & Nein & Nein & [Wind01] \\
\hline & DASCh & FP & Nein & Nein & Nein & Nein & [PaVa98] \\
\hline
\end{tabular}

Tabelle 2: Ausgewählte MAS-Architekturen zur Effektivierung von Supply Networks

Die in der Literatur vorgestellten agentenbasierten Ansätze zur Effektivierung des SCEM unterstützen zumeist nur isolierte Teilbereiche eines Supply Network. Ansätze, die auf das gesamte Supply Network fokussieren sind seltener zu finden. Zudem handelt es sich zumeist um Rahmenarchitekturen bzw. Forschungsprototypen, die sich noch nicht im Praxiseinsatz (wie bspw. das MAS CoagenS) befinden. Multimodale Eingabegeräte, insbesondere ein sprachbasierter Zugriff, sowie agentenbasiertes $\mathrm{T} \& \mathrm{~T}$ auf der Basis von RFID oder die Integration von Web Services zum automatischen Datenaustausch werden von den derzeitigen MAS zumeist noch nicht unterstützt (vgl. Tabelle 2).

Der Stand auf dem Weg zur Realisierung ubiquitärer Supply Networks auf der Basis der Agententechnologie lässt sich somit derzeit (Stand: Oktober 2004) wie folgt zusammfassend charakterisieren:

- Isolierte Teilbereiche: Bei den bisher im Bereich des SCEM zum Einsatz kommenden agentenbasierten Systemen handelt es sich zumeist um zentralisierte bzw. isolierte und monolithische Systeme, die auf die Optimierung der Geschäftsprozesse nur eines Unternehmens innerhalb des Supply Network beschränkt sind bzw. nur Teilbereiche des SCEM adressieren. Innerhalb des $A$ gent.Enterprise-Szenarios $\left[\mathrm{Frey}^{+} 03\right]$ wird aus diesem Grund in einem Ver- 
bundprojekt eine Architektur entwickelt, die eine Integration verschiedener MAS im Bereich des SCEM ermöglicht. Agent.Enterprise umfasst die MAS IntaPS, KRASH und FABMAS im Bereich der Produktionslogistik, ATT/SCC zum Tracking sowie DISPOWEB zur unternehmensübergreifenden Koordination.

- Adaptivität: Agentensysteme, die eine automatische Rückkopplung, d. h. eine kontinuierliche Bewertung getroffener Entscheidungen und Auswertung vergangener Daten als Grundvoraussetzung für die Realisierung adaptiver Supply Networks unterstützen, stehen erst am Anfang.

- Dynamik: Die heutigen Agentensysteme im SCEM sind noch zu statisch. Da es sich bei Supply Networks um „lebende“ Organisationsstrukturen handelt, sind MAS im SCM zu entwickeln, die wie „lebende Organismen“ sich selbst rekonfigurieren können. Grafische Modellierungswerkzeuge zur benutzerfreundlichen Abbildung und Rekonfiguration adaptiver bzw. ubiquitärer Supply Networks wären hierbei von Vorteil und wünschenswert.

- Akzeptanzforschung: Derzeit liegen noch kaum Erfahrungswerte aus dem täglichen Praxiseinsatz bzw. zur Praxistauglichkeit von MAS zur Effektivierung von Supply Networks vor. Aufgrund hoher Sicherheitsanforderungen und dem Zugriff auf sensible Unternehmensdaten durch Agenten fehlt in der Praxis oftmals das notwendige Vertrauen in den Einsatz von MAS im SCEM.

- Grad der Kooperation: Ein MAS im SCEM sollte die Möglichkeit bieten, den Datenzugriff sowie die Verhandlungstaktik in Abhängigkeit von der Kooperationsbereitschaft bzw. dem Vertrauen zum jeweiligen Netzwerk-Partner individuell zu regeln. Entsprechende Verhandlungstaktiken, insbesondere zur Unterstützung multiattributiver, multilateraler Verhandlungen, werden von den derzeitigen MAS im SCEM jedoch noch nicht unterstützt.

- Mobiler, multimodaler Zugriff: Durch den Einsatz mobiler Endgeräte ergeben sich neue Anwendungsbereiche. Bspw. auf Baustellen, in schlecht beleuchteten Lagerhallen sowie im Straßenverkehr ist es von Vorteil, wenn wichtige Informationen auch sprachbasiert abgerufen werden können und nicht umständlich über eine kleine Handy-Tastatur. Der PDA mit integrierter Kamera und RFID-Reader eines Auslieferungsfahrers kann bspw. beschädigte Ware direkt aufnehmen, durch die RFID-Technologie automatisch identifizieren und die Schadensmeldung (inkl. Videosequenz zur Schadenserfassung) per Mobilfunk zur weiteren Verarbeitung an das MAS senden.

\section{Rahmenarchitektur CoS.MA}

In diesem Abschnitt wird die Rahmenarchitektur des MAS CoS.MA (= Cooperative and Ubiquitous $\boldsymbol{S}$ upply-Network $\boldsymbol{M}$ onitoring $\boldsymbol{A}$ gents) zur Realisierung ubiquitärer Supply Networks (vgl. Tabelle 1) vorgestellt. 


\section{$5.1 \quad$ Zielsetzung}

Mit CoS.MA wird das Hauptziel verfolgt, die Effizienz des gesamten Supply Network zu steigern, so dass prinzipiell alle Partner des Supply Network Wettbewerbsvorteile erlangen können. Das MAS CoS.MA soll den Mitgliedern des Supply Network Handlungsempfehlungen, insbesondere zu den folgenden Fragestellungen der Beschaffungs-, Produktions-, und Distributions-Logistik geben können:

- Wie kann ich trotz momentaner Lieferengpässe meines Hauptlieferanten die Nachfrage erfüllen?

- Wie kann ich trotz meiner niedrigen Lagerbestände in angemessener Weise auf den kurzfristigen Nachfrageanstieg meines Hauptabnehmers reagieren?

- Wie kann ich die Durchlaufzeiten von Produkt A innerhalb des Supply Network weiter verkürzen?

- Wie kann ich gemeinsam mit meinen Zulieferern die Produktionsplanung von Produkt B aufgrund momentaner Fertigungsengpässe kurzfristig ändern?

- Wie kann ich die Auslastung meiner Auslieferungsfahrzeuge erhöhen und Leerfahrten vermeiden?

- In welchem Auftragsstatus befindet sich momentan Produkt C?

Die zum Teil sehr heterogen gestalteten PPS-/ERP-/SCM-Systeme der in einem Supply Network beteiligten Akteure sollen durch CoS.MA dem Ziel einer unternehmensübergreifenden Informationsverarbeitung entlang des Supply Network näher gebracht werden sowie sinnvoll in die CoS.MA-Rahmenarchitektur zur Effektivierung des SCEM integriert werden. CoS.MA soll daher auf Planungsalgorithmen und Daten der bereits in den Unternehmen eingesetzten PPS-/SCMsowie ERP-Systeme zurückgreifen können.

Die Mitglieder eines Supply Network können durch CoS.MA mit den benötigten Informationen versorgt werden, so dass bspw. jederzeit von jedem Ort ersichtlich ist, wo welche Bestände, Produkte, oder Auslieferungsfahrzeuge vorhanden sind. Hauptziel ist es hierbei, schnell auf veränderte Bedingungen innerhalb des Supply Network reagieren zu können und sowohl die Entscheidungsträger in den Unternehmen als auch mobile Mitarbeiter (Außendienstmitarbeiter, Baustellenleiter, Auslieferungsfahrer, etc.) mit den für ein erfolgreiches Wirtschaften innerhalb des Supply Network notwendigen Informationen zu versorgen.

\subsection{Anforderungen}

Da in der betrieblichen Realität nicht von statischen sondern nur von temporär statischen Supply Networks ausgegangen werden kann, ist es notwendig, ein offenes, adaptives und dynamisches MAS zu entwickeln. 
Mit der Rahmernarchitektur CoS.MA wird ein dezentraler Planungsansatz verfolgt, da hierdurch flexiblere Reaktionszeiten im Hinblick auf unvorhergesehene Ereignisse bzw. Störungen innerhalb des Netzwerks erzielt werden können. Der lokale Planungsaufwand ist bei verteilter Planung zudem geringer als bei zentraler Planung [ $\mathrm{Grol}^{+} 01, \mathrm{~S} .143 \mathrm{f}$ ]. Ein dezentraler Ansatz entspricht darüber hinaus dem natürlichen Bedürfnis einzelner Unternehmen nach Unabhängigkeit und Entscheidungsautonomie. Die Bereitschaft, unternehmensinterne Daten ungefiltert an ein dominantes Unternehmen weiterzuleiten ist eher begrenzt. Eine zentrale Koordination erfordert zudem einen immensen Datenerfassungs- und Übertragungsaufwand. Darüber hinaus sind Störfälle innerhalb des Supply Network eher in kollaborativer Zusammenarbeit und unter Einsatz dezentraler Koordinationsformen zu lösen. Jedes Unternehmen des Supply Network soll daher ein lokales CoS.MA-MAS zum Initialisieren von Agenten erhalten, das andere Agenten wiederum aufnehmen kann oder deren Agenten wiederum zu anderen Agentenplattformen migrieren kann, um relevante Planungsdaten einzuholen. Der Ausfall einer Agentenplattform innerhalb eines Supply Network wäre letztendlich weniger kritisch als der Ausfall einer zentralen Agentenplattform, die den gesamten Informationsaustausch koordiniert.

Weitere wichtige Anforderungen an das MAS sind die Skalierbarkeit sowie Robustheit, d. h. das MAS muss beliebig erweitert werden können, wenn neue Mitglieder in das Netzwerk aufgenommen werden sollen. Des Weiteren müssen jederzeit auch Mitglieder wieder ausgeschlossen werden können.

\subsection{Rahmenarchitektur}

Das Multi-Agenten-System CoS.MA soll mit den vorhandenen Enterprise Resource Planning (ERP-)Systemen und SCM-Systemen der beteiligten Unternehmen interagieren und relevante Planungsinformationen wie z. B. Stammdaten einzelner Produkte, Fertigungsstrukturdaten sowie prognostizierte Absatzdaten austauschen können. Daten vorhandener ERP-Systeme können dadurch zur Planung, Optimierung und Informationsversorgung herangezogen werden. CoS.MA setzt somit auf die bestehende IT-Landschaft auf. Die Implementierung soll auf Basis einer FIPA-konformen Agentenplattform (JADE) erfolgen. Als Schnittstellen zum Informationsaustausch sollen FIPA-ACL, XML, PML, Web-EDI, VDA, (Mobile) Web Services, SQL sowie ODBC dienen. Die Multi-Agenten-Systeme der beteiligten Uunternehmen können somit auch unternehmensübergreifend Daten aus den verschiedenen ERP-Systemen zur Generierung von Handlungsempfehlungen nutzen.

Die MAS-Rahmenarchitektur (vgl. Abbildung 3) ist in Form eines Peer-to-Peer (P2P)-Netzwerks aufgebaut, und beliebig erweiterbar, d. h. sukzessive können neu hinzukommende Lieferanten, Produzenten, Abnehmer oder 3rd Party Logistics Provider integriert werden. Die Grundidee des P2P-Ansatzes ist eine dezentrale 
Netzwerkstruktur zwischen flexibel und direkt miteinander verbundenen Knoten [ScFi02]. Die Knotenpunkte sollen als gleichberechtigte Partner (Peers) die Netzwerkstruktur zur Duchführung kollaborativer und adaptiver Prozesse bilden. Die einzelnen Knoten des P2P-Netzwerks (d.h. die einzelnen CoS.MA-Agentenplattformen) sind dabei sowohl in der Lage Server- als auch Client-Funktionalität zu leisten.

Agententechnologie und P2P-Ansatz können in der CoS.MA-Rahmenarchitektur wechselseitig voneinander profitieren: Durch den verfolgten P2P-Ansatz mit offenen XML-basierten Standards sowie Web Services zum Informationsaustausch entsteht ein dynamisches und flexibles Netzwerk, das trotz seiner Komplexität aufgrund seiner Dezentralität für die einzelnen Unternehmen noch administrierbar bleibt. Daten können individuell vor Zugriff gesichert werden (je nach Kooperationsbereitschaft), so dass sensible Firmendaten von den Agenten anderer Unternehmen nur sofern erwünscht einsehbar sind. Der Zugriff auf CoS.MA soll sowohl über Web-Browser als auch über multimodale mobile Endgeräte und insbesondere auch sprachbasiert (VoiceXML) erfolgen (vgl. Abbildung 3).

Um den Überwachungs- und Kommunikationsaufwand einzugrenzen, sollen gezielt diejenigen Aufträge und potentiellen Störereignisse entlang des Supply Network überwacht werden, die aufgrund historischer Erfahrungswerte und Experteninterviews als potentiell kritisch eingestuft werden müssen. Hierzu werden zur Klassifizierung der potentiell gefährdeten Aufträge und Störereignisse „Risikoprofile“ [ZiPa03] erstellt (vgl. Abbildung 3). Bestehende Risikoprofile können durch Rückkopplung und Analyse historischer Daten (Tracing) automatisch und kontinuierlich an sich verändernde Störungsschwerpunkte innerhalb des Supply Network angepasst werden.

CoS.MA überprüft auf der Basis der Risiko- und Personenprofile, ob Entscheidungsträger benachrichtigt sowie Workflows angestoßen werden müssen. Zu unterscheiden ist zwischen geplanten Ereignissen wie z. B. Eingang einer Ware am Lager, Rückmeldung vom Spediteur bei Auslieferung einer Ware, Standortmeldungen, Messwertmeldungen sowie ungeplanten Ereignissen wie z. B. Schadensmeldungen oder Unfälle. Letztere sind als kritisch anzusehen. Über geplante Ereignisse sollen die Agenten nur dann informieren, sofern dies in den Personenprofilen der Entscheidungsträger als gewünscht hinterlegt wurde. Ein ContentKonvertierer (vgl. Abbildung 3) soll auf Basis von XSL(T) helfen, die Informationen in die Auszeichnungssprachen (HTML, VoiceXML, WML, dHTML, etc.) der jeweiligen (mobilen) Endgeräte der Entscheidungsträger zu konvertieren. 


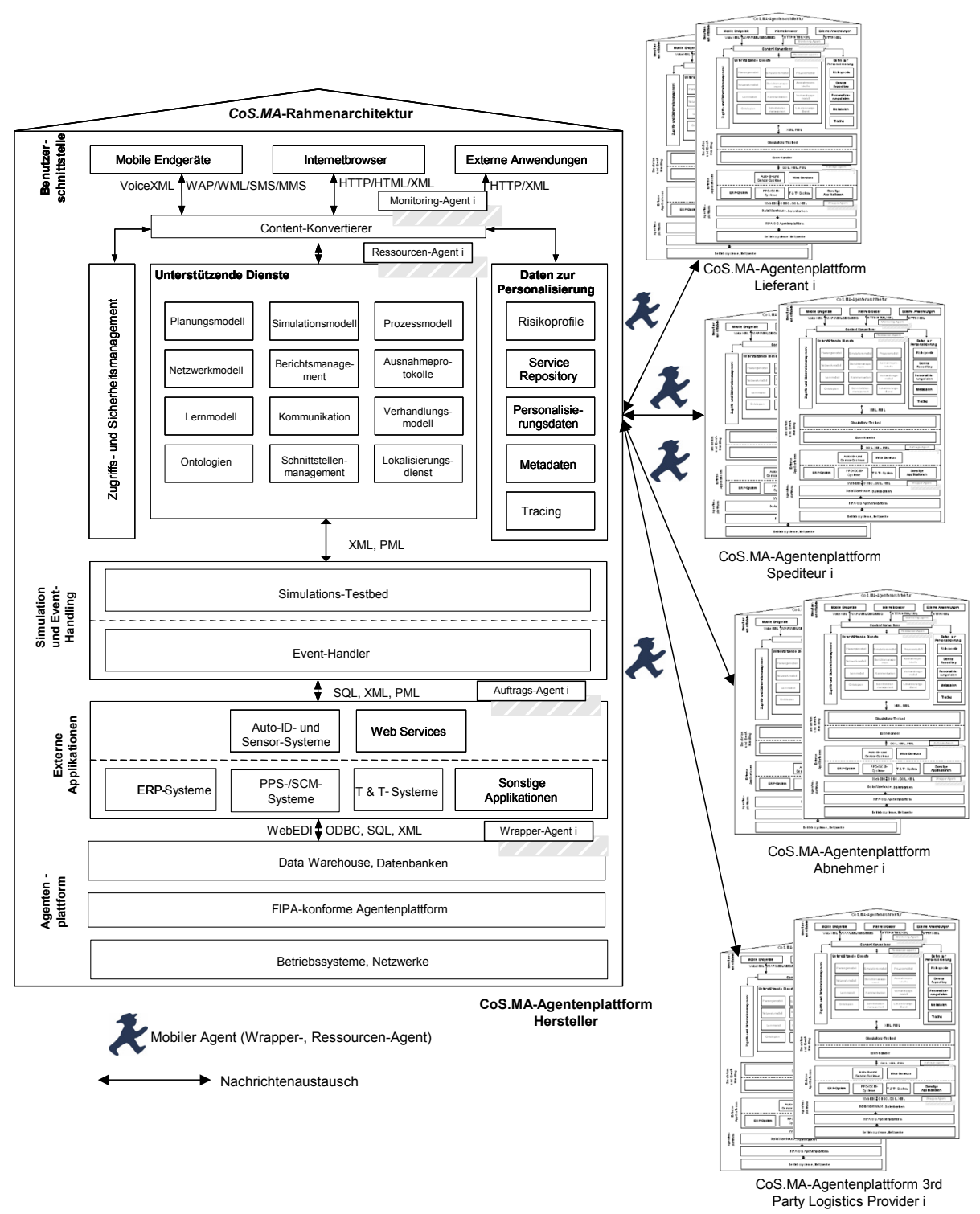

Abbildung 3: CoS.MA -Rahmenarchitektur 


\subsection{Agentenrollen}

Die CoS.MA-Rahmenarchitektur umfasst sowohl stationäre als auch mobile, in Java implementierte Agenten, die zu den Quellen der von ihnen benötigten Informationen sowie den mobilen Endgeräten der Entscheidungsträger entlang des Supply Network migrieren können. Die Agenten repräsentieren dabei organisatorische Einheiten auf unterschiedlichen Hierarchieebenen wie z. B. Unternehmensverbund, Unternehmen, Werk, Lager, Arbeitsplätze, Maschinen, Transportaufträge oder Auslieferungsfahrzeuge, d. h. Entitäten des Supply Network, die zur Effektivierung eines Supply Networks als planungsrelevante Grundlage für Handlungsempfehlungen dienen. Jeder Agent soll die zu einem bestimmten Zeitpunkt vorhandenen Informationen, mit Ausnahme der von Unternehmen zum Zugriff durch Agenten gesperrten Informationsbestände, im gesamten Netzwerkverbund einholen können.

Alle Agenten sind lernfähig und erhalten Rückkopplung zu den von ihnen getroffenen Entscheidungen. Die Lernfähigkeit der Agenten soll eine ständige Anpassung an den Wettbewerb der durch die Agenten repräsentierten Organisationseinheiten sowie deren Beziehungsgeflechten innerhalb des Supply Network ermöglichen. Jeder Agent soll über eine Rückkopplung der erzielten Planungsergebnisse lernen, indem bspw. Entscheidungsträger ein kurzes Feedback zu erteilten Handlungsempfehlungen geben und auf diese Weise Soll-Ist-Vergleiche durch die Agenten durchgeführt werden können. Mittels Tracing gesammelte und verdichtete Daten sollen als zusätzliche Planungsgrundlage dienen. Auf der Basis dieser Daten können dann Algorithmen eingesetzt werden, die ein Lernen der beteiligten Agenten durch Rückkopplung ermöglichen und helfen, ein adaptives Kompetenznetzwerk zwischen den Beteiligten des Supply Network aufzubauen.

Im Netzwerkmodell, einer der unterstützenden Dienste von CoS.MA, wird das gesamte inner- und zwischenbetriebliche Supply Network abgebildet; es dient damit jedem Agenten als Hilfe (sog. ,Yellow-Pages“), den richtigen Agenten der jeweils nachgelagerten Stufe finden und kontaktieren zu können. Die in CoS.MA integrierte Simulations-Testumgebung (vgl. Abbildung 3) soll es den Agenten erlauben, noch bevor den Entscheidungsträgern (Produktionsplaner, Auslieferungsfahrer, etc.) konkrete Handlungsempfehlungen erteilt werden, für unerprobte Störereignisse Maßnahme-Ergebnis-Kombinationen in Form von Vorab-Simulationen „durchspielen“ zu können.

Folgende Agentenrollen sind in CoS.MA vorgesehen:

- Monitoring-Agenten: Handeln proaktiv und überwachen kritische Größen entlang des Supply Network. Entscheidungsträger können Monitoring-Agenten initialisieren und angeben, in welcher Form und über welche (mobilen) Endgeräte sie beim Unter- oder Überschreiten bestimmter Werte alarmiert werden wollen. 
- Auftrags-Agenten: Auftrags-Agenten geben Auskunft über den Stand der Aufträge entlang des Supply-Network.

- Ressourcen-Agenten: Agenten mit Verhandlungskompetenz, die im Auftrag der Disponenten von Ressourcen wie z. B. Unternehmenseinheiten, Lagerhallen, Arbeitsplätzen, Maschinen, Auslieferungsfahrzeugen, etc. im Supply Network mit anderen Ressourcen-Agenten verhandeln.

- Wrapper-Agenten: Extrahieren relevante Informationen aus den Datenbanken der Netzwerk-Mitglieder.

\subsection{Nachrichtenkommunikation in $\operatorname{CoS} . M A$}

Für die Agentenkommunikation ist ein standardisiertes Nachrichtenformat erforderlich. Hierzu ist die Agentenkommunikationssprache FIPA-ACL vorgesehen, deren wesentliche Potentiale in einer hohen Verbreitung sowie ihrer Erweiterbarkeit liegen [FIPA04]. Verhandlungen sollen in CoS.MA auf der Basis von XML-Standards, eingebettet in das FIPA-ACL-Nachrichtenformat, sowie Web Service-Technologien erfolgen. Abbildung 4 stellt exemplarisch die XML-basierte Physical Markup Language PML integriert in das FIPA-ACL Nachrichtenformat dar. Im Beispiel aus Abbildung 4 informiert ein Ressourcen-Agent einen Auftrags-Agenten über das Ereignis, dass eine Palette an einem bestimmten Sensor um 5 Uhr 44 am 06. Juni 2004 (vgl. Abbildung 4, Timestamp-Tag) am Hafenlager-Tor 15 bei Unternehmen A1 in Hamburg lokalisiert wurde.

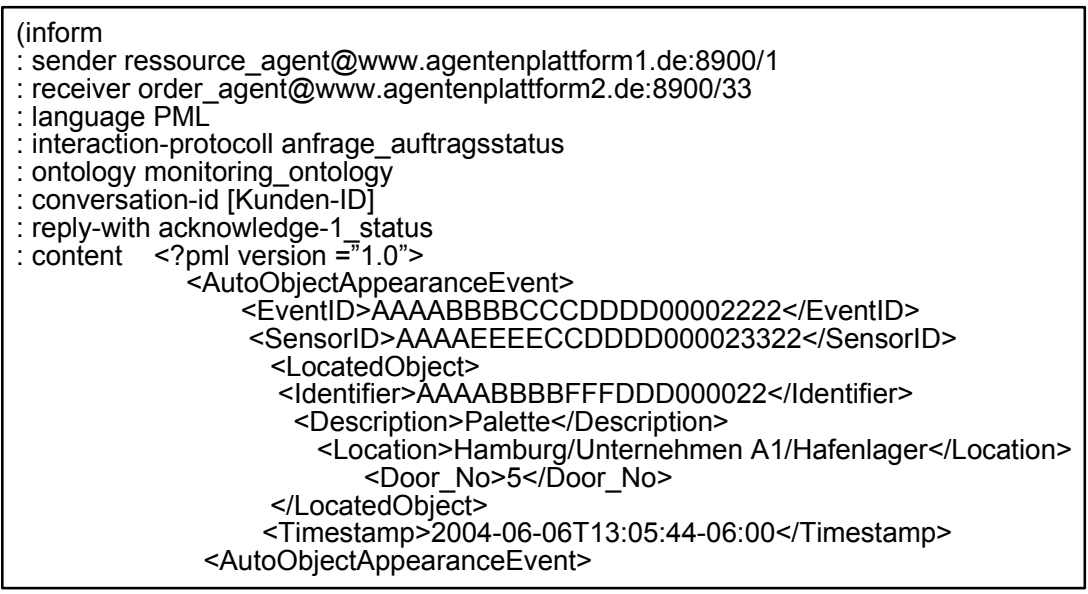

Abbildung 4: FIPA-ACL-Template mit PML-Nachricht 


\subsection{Nutzenpotentiale von $\operatorname{CoS} . M A$}

Mit der Implementierung von CoS.MA werden potentiell die nachfolgenden Nutzeffekte anvisiert:

- Mehr Informationstransparenz: Die Prozessbeteiligten können auf aktuelle und genaue Informationen zu den Logistikprozessen in Realzeit zugreifen.

- Höhere Qualität der Leistungsprozesse: Durch beschleunigte Prozesse kann eine bessere Kundenbetreuung erzielt werden. Dienste können auch auf mobile Entscheidungsträger ausgeweitet werden.

- Adaption und kontinuierliche Optimierung: Auf Basis der vorgesehenen Rückkopplungsmechanismen und des Monitoring prozessbezogener Kennzahlen können Logistikprozesse kontinuierlich optimiert werden.

- Entlastung der Mitarbeiter von Routinetätigkeiten: Durch die automatisierte Überwachung von Logistikprozessen auf Basis der Auto-ID-Technologien und Erfassung von Daten können in vielen Bereichen die manuelle Eingabe von Problemfällen und Störereignissen entfallen.

Die im Rahmen dieses Beitrags vorgestellte CoS.MA-Rahmenarchitektur erweitert derzeitige MAS-Architekturen zur Steuerung adaptiver Supply Networks wie PAMAS, Macroscope und CoagenS (vgl. Tabelle 2), um Web Services zum automatisierten Datenaustausch sowie multimodale Zugriffsmöglichkeiten (WebBrowser, mobile Engeräte, Voice-Zugriff) zur Realisierung und Überwachung ubiquitärer Supply Networks. Mit CoS.MA wird darüber hinaus das Ziel verfolgt, agentenbasiertes Tracking und Tracing mit Hilfe der Auto-ID-Technologie RFID sowie Sensor-Technologien zu unterstützen.

\section{CoS.MA-Simulations-Prototyp}

Da es in der Erprobungsphase des CoS.MA-MAS noch nicht möglich ist, auf laufende ERP-, PPS- sowie SCM-Systeme im Praxiseinsatz zuzugreifen, wird aufgrund der Komplexität des MAS ein Simulationsansatz verfolgt. Simulationen sollen dabei mit dem Ziel, Praxistauglichkeit, Kommunikationsaufwand und Agilität des MAS zu ermitteln anhand realitätsnaher Testdaten und Anwendungsszenarien erfolgen. Zur Durchführung der Simulationen soll zunächst ein elementares Simulationsszenario mit einer Netwerkstruktur von 3 CoS.MA-Systemen, die jeweils Zugriff auf ERP-Systeme (SAP R/3 sowie Infor:COM) haben, realisiert und sukzessive ausgebaut werden (vgl. Abbildung 5). Es ist vorgesehen, die CoS.MAAgentenplattformen in der Simulationsstudie auf 2 SAP R/3-Systeme sowie ein infor:com-ERP-System zugreifen zu lassen, die die Datenbestände von zunächst 3 Unternehmen (Lieferant, Abnehmer und Speditionsunternehmen) simulieren. Die Simulationsdaten werden mit Hilfe eines Simulations-Tools automatisch generiert und im Zeitablauf fortgeschrieben. Der Zugriff wird multimodal (mobile Endge- 
räte, Voice, Web Browser) erfolgen. Als Machbarkeitsstudie soll die in Abschnitt 3.2 beschriebene Auto-ID-Infrastruktur ebenfalls in die Netzwerkarchitektur des CoS.MA-Simulations-Prototyps integriert werden und zur Simulation eines T \& T von fiktiven Produkten dienen. Die einzelnen Systeme werden mittels eines Hub sowie einem Wireless Access Point für die mobilen Endgeräte zu einem Local Area Network integriert (vgl. Abbildung 5).

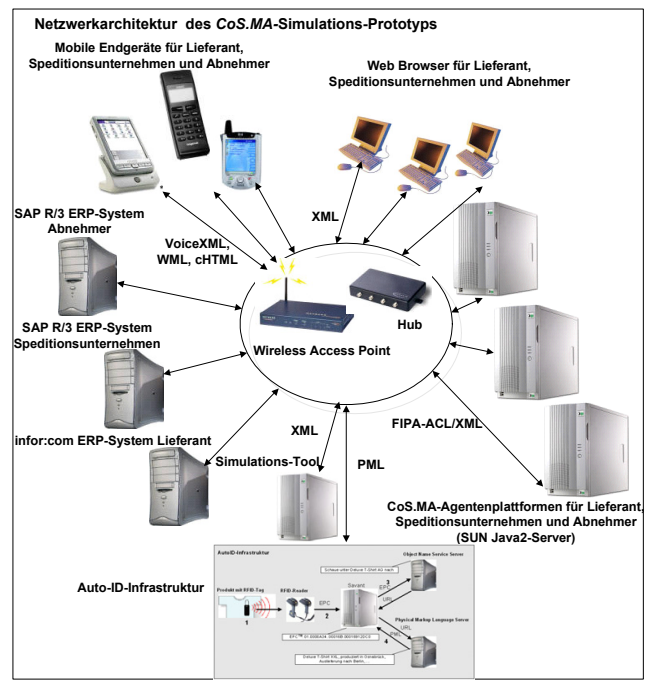

Abbildung 5: Netzwerkstruktur des CoS.MA-Simulations-Prototyps

\section{Zusammenfassung und Ausblick}

In dem vorliegenden Beitrag, der im Rahmen des vom BMBF geförderten Verbundprojekts "Geschäftsprozesse und Nutzerschnittstellen im Mobile Business auf Basis des Wireless Internet" entstand, wurde der Begriff des „Ubiquitären Supply Network" neu eingeführt und als weitere Entwicklungsstufe aufbauend auf adaptiven Supply Networks beschrieben. Der Status Quo zum Einsatz der Agententechnologie zur Effektivierung des SCEM wurde untersucht. Insbesondere im Hinblick auf Sicherheitsaspekte, Standardisierungen zum automatisch interpretierbareren Datenaustausch zwischen heterogenen Systemen im SCEM sowie der Integration von Auto-ID- und Mobile Computing-Technologien zeigen sich noch erheblicher Forschungsbedarf und Entwicklungspotentiale. Die im Rahmen des vorliegenden Beitrags vorgestellte Rahmenarchitektur CoS.MA soll Abhilfe schaffen und ist ein erster Schritt zur Realisierung ubiquitärer Supply Networks. Derzeit erfolgt die Implementierung der in UML spezifizierten Rahmenarchitektur CoS.MA. Es ist geplant, das MAS CoS.MA nach der Implementierung und Simulation in Kooperation mit Praxispartnern mittels Realdaten zu analysieren. 


\section{Literatur}

[Bode ${ }^{+}$01] Bodendorf, F.; Butscher, R.; Zimmermann, R.: Konzept zur agentengestützten Auftragsüberwachung in Supply-Chains. In: Logistik Management 2/3. 2001, S. 13-24.

[ChMa96] Chavez, A.; Maes, P.: Kasbah: An Agent Marketplace for Buying and Selling Goods. In: Crabtree, B.; Jennings, N. (Eds.): Proc. of the First International Conference on the Practical Application of Intelligent Agents and Multi-Agent Technology (PAAM '96). The Practical Application Company Ltd: Blackpool, 1996, S. 75-90.

[Dang ${ }^{+}$02b] Dangelmaier, W.; Gajewski, T.; Pape, U.; Rüther, M.: Supply Chain Management am Beispiel des Projektes CoagenS. In: Loos, P.; Gronau, N. (Hrsg.): E-Business - Integration industrieller ERP-Architekturen. Cuvillier Verlag: Göttingen, 2002, S. 5972.

[Dang ${ }^{+}$04] Dangelmaier, W.; Pape, U.; Rüther, M.: Agentensysteme für das Supply Chain Management: Grundlagen, Konzepte, Anwendungen. Deutscher Universitäts-Verlag: Wiesbaden, 2004.

[Falk95] Falk, J.: Ein Multi-Agentensystem zur Transportplanung und -steuerung bei Speditionen mit Trampverkehr, Vol. 102. Dissertationen zur Künstlichen Intelligenz. Akademische Verlagsgesellschaft: Berlin, 1995.

[Ferb99] Ferber, J.: Multi-Agent Systems: An Introduction to Distributed Artificial Intelligence. New York, 1999.

[FIPA04] FIPA: Foundation for Intelligent Physical Agents. http://www.fipa.org/, Abruf am 2004-06-22.

[Fisc ${ }^{+}$95] Fischer, K.; Müller, J. P.; Pischel, M.; Schier, D.: A Model for Cooperative Transportation Scheduling. In: Proceedings of the First International Conference on Multi-Agent-Systems (ICMAS 95). San Francisco, 1995, S. 109-116.

[Floe ${ }^{+}$03] Floerkemeier, C.; Anarkat, D.; Osinski, T.; Harrison; M.: PML Core Specification 1.0: Auto-ID Center Recommendation, $15^{\text {th }}$ of September 2003. http://www.epcglobalinc.org/standards_technology/Secure/v1.0/PML_Core_Specification_v1.0.pdf, Abruf am 2004-06-22.

[Främ ${ }^{+}$03] Främling, K.; Holmström, J.; Ala-Risku, T.; Kärkkäinen, M.: Product Agents for Handling Informationen about Physical Objects; Research Report, Helsinki University of Technology. http://www.cs.hut.fi/Publication/Reports/B153.pdf, Abruf am 2004-0622.

[Frey ${ }^{+}$03] Frey, D.; Mönch, L.; Stockheim, T.; Woelk, P.; Zimmermann, R.: Agent.Enterprise - Integriertes Supply Chain Management mit hierarchisch vernetzten Multiagenten-Systemen. In: GI (Hrsg.): Tagungsband der GI-Jahrestagung 2003, Frankfurt, 2003.

[Grol ${ }^{+}$01] Grolik, S.; Stockheim, T.; Wendt, O.; Albayrak, S.; Fricke, S.: Dispositive Supply Web-Koordination durch Multiagentensysteme. In: Wirtschaftsinformatik 2, 2001. S. $143-155$. 
[Hens98] Henseler, H.: Aktive Ablaufplanung mit Multi-Agenten, Vol. 180. Dissertationen zur Künstlichen Intelligenz, Akademische Verlagsgesellschaft: Berlin, 1998.

[KeKr04] Keller, S.; Krol, B.: E-Logistics und Bullwhip-Effekt. In: WISU 33, 2004. S. 308-312.

[Lang02] Langley Jr., C. J.; Allen, G. R.; Tyndall, G. R.: Third-Party Logistics Study - Results and Findings of the 2002 Seventh Annual Study. http://tli.isye.gatech.edu/ downloads/3PLStudy_2002.pdf, 2002, Abruf am 2004-06-22.

[Maes95] Maes, P.: Artificial Life meets Entertainment: Interacting with Lifelike Autonomous Agents. In: Communications of the ACM 11, 1995. S. 108 -114.

[Mann95] Mannmeusel, T.: Ein verteilter Ansatz zur operativen Produktionsplanung auf der Basis eines Multi-Agenten-Systems. In: Klauck, C.; Müller, J. (Hrsg.): Künstliche Intelligenz \& Verteilte PPS-Systeme, Bericht Nr. 5/95, Universität Bremen, 1995.

[Melo03] Meloan, S.: Toward a Global "Internet of Things", $11^{\text {th }}$ of Nov. 2003; http://java.sun.com/developer/technicalArticles/Ecommerce/, Abruf am 2004-06-22.

[Niss02] Nissen, V.: Supply Chain Event Management. In: Wirtschaftsinformatik 5, 2002. S. $477-480$.

[PaVa98] Van Parunak, H.; VanderBok, R. S.: Modeling the Extended Supply Network, Working paper, Industrial Technology Institute, Ann Arbor, USA, 1998. http://www.agent.ai/doc/upload/200302/paru98_2.pdf, Abruf am 2004-06-22.

[SAP02] SAP: SAP White Paper: Adaptive Supply Chain Networks. http://www.sap.com/ solutions/scm/adaptivesupplychain.asp, 2002, Abruf am 2004-06-22.

[ScFi02] Schoder, D.; Fischbach, K.: Peer-to-Peer: Anwendungsbereiche und Herausforderungen. In: Schoder, D.; Fischbach, K.; Teichman, R. (Hrsg.): Peer-to-Peer. Springer: Heidelberg, 2002, S. 4-23.

[ScHö03] Scholz-Reiter, B.; Höhns, H: Integrated software agents: enabling technology for collaborative E-logistics and E-business. In: International Journal of Computer Integrated Manufacturing 7-8. 2003. S. 517-525.

[Szir02] Szirbik, N.: A Negotiation Enabling Agent Based Infrastructure: Composition and Behavior. In: Information Systems Frontiers, Volume 4, Issue 1. 2002. S. 85-99.

[Tsve ${ }^{+}$97] Tsvetovatyy, M.; Gini, M.; Mobasher, B.; Wieckowski, Z.: MAGMA: An agentbased virtual market for electronic commerce. In: Applied Artificial Intelligence 6, 1997. S. 501-524.

[Wind01] Windischbauer, T.: Kollaborative Supply-Chain-Execution in heterarchisch koordinierten Logistiknetzwerken auf der Basis von mobilen Agentensystemen. Linz, 2001.

[Woo199] Wooldridge, M.: Intelligent Agents. In: Weiss, G. (Ed.): Multiagent Systems: A Modern Approach to Distributed Artificial Intelligence. Cambridge, 1999, S. 25-76.

[ZiPa03] Zimmermann, R.; Paschke, A.: PAMAS - an Agent-based Supply Chain Event Management System. In: Proc. of Americas Conf. on Information Systems 2003. http://ibis.in.tum.de/staff/paschke/docs/PAMAS_AMCIS_03.pdf, 2003, Abruf am 2004-06-22. 